\title{
Painful fingertip swelling of the long finger
}

\author{
Fabio Becce • Biljana Jovanovic • Louis Guillou • \\ Nicolas Theumann
}

Received: 22 March 2011 / Revised: 15 May 2011 / Accepted: 20 May 2011 /Published online: 18 June 2011

(C) ISS 2011

\section{Part I}

\begin{abstract}
A 35-year-old Caucasian woman presented with a 14-month history of relapsing painful fingertip swelling of the left middle finger observed after a minor trauma. Conventional radiographs (Fig. 1) were performed at presentation. After failure of conservative treatment, the patient underwent initial surgery without success. Follow-up MRI (Fig. 2) and CT (Fig. 3) were obtained at our institution 6 months after the first surgical procedure (Figs. 1a-b, 2a-d, 3 and 4).
\end{abstract}

The diagnosis can be found at doi:10.1007/s00256-011-1219-y.

F. Becce $\cdot N$. Theumann $(\square)$

Department of Diagnostic and Interventional Radiology,

Centre Hospitalier Universitaire Vaudois, University of Lausanne,

Rue du Bugnon 46,

1011 Lausanne, Switzerland

e-mail: nicolas.theumann@chuv.ch

B. Jovanovic

Plastic and Hand Surgery Centre,

1003 Lausanne, Switzerland

L. Guillou

University Institute of Pathology,

Centre Hospitalier Universitaire Vaudois,

1011 Lausanne, Switzerland
Funding None

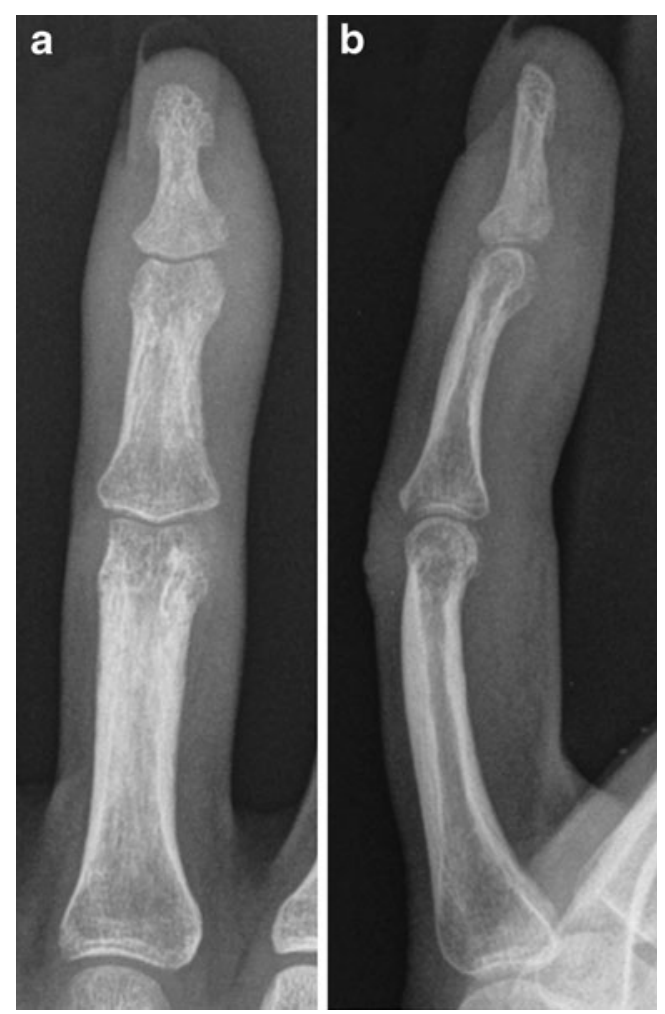

Fig. 1 Posteroanterior (a) and lateral (b) radiographs of the left long finger 
Fig. 2 Sagittal (a) and axial (b) fat-suppressed gadoliniumenhanced T1-weighted turbo spin-echo MR images of the left long finger (right side of the picture in b). Four-dimensional contrast-enhanced MR angiographic images of the left hand in the arterial (c) and venous (d) phase
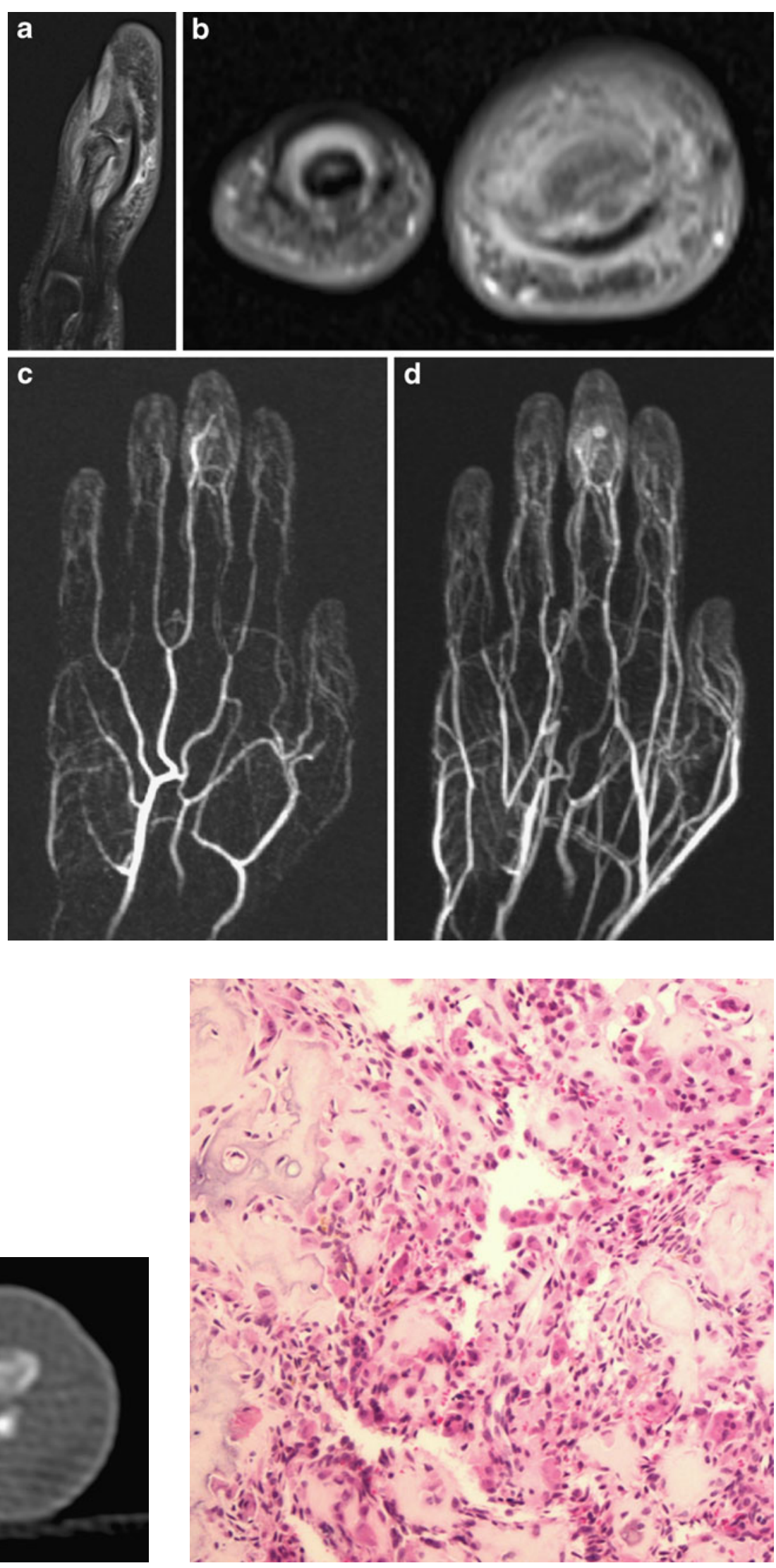

Fig. 4 Histopathology image (hematoxylin and eosin stain (H\&E); original magnification $\times 200$ )
Fig. 3 Axial unenhanced CT image of the left middle (right side of the picture) and ring fingers 